\title{
Fractografía y disipación de energía cinética en un panel balístico de cerámica/polímero sometido al impacto de un proyectil metálico
}

\author{
Fractography and dissipation of kinetic energy in \\ a polymeric/ceramics ballistic panel submitted to \\ the impact of a metallic projectile
}

Giovanni Barrera Torres ${ }^{1}$, Juan Manuel Vélez Restrepo ${ }^{2}$

\author{
${ }^{1}$ Instituto Tecnológico Metropolitano. Medellín, Colombia \\ e-mail: giovannibarrera@itm.edu.co \\ ${ }^{2}$ Universidad Nacional de Colombia, Sede Medellín. Facultad de Minas. Colombia \\ e-mail: imvelez@unal.edu.co
}

\section{RESUMEN}

En este trabajo se exploró el comportamiento y los mecanismos de daño de un panel laminado compuesto a partir de materiales poliméricos y cerámicos, ante el impacto de un proyectil metálico. Se realizó el diseño de un sistema con la cara de impacto en material cerámico ( $\mathrm{SiC}$ ), con respaldo elaborado en polietileno de peso molecular ultra alto (UHMWPE), placas elaboradas con geometría cuadrada de $200 \mathrm{~mm}$ de lado y $5 \mathrm{~mm}$ de espesor, y cortes de tejido polimérico con iguales dimensiones de área. Se tomó como referencia el montaje de la norma (NIJ) Standard 0101.06 para el ensayo a impacto balístico; en el material cerámico se identificó la formación de cráter, fracturas y delaminación como mecanismos de falla predominantes, utilizando microscopia óptica y de barrido; en el material polimérico se observó fibrilación, torsión, fusión de fibras, delaminación y deformación cónica y posteriormente se analizaron las muestras empleando los modelos de balance de energía cinética de "Morye, Smith y de Retch e Ipson". El resultado evidenció que el arreglo con mayor influencia para disipar energía, es el panel elaborado con doble placa cerámica (SiC) y doble capa de tejido polimérico como respaldo.

Palabras clave: sistemas compuestos, impacto, mecanismos de falla, disipación de energía cinética.

\section{ABSTRACT}

In this study we have explored the behavior and the damage mechanisms of a laminate panel composed of polymeric and ceramic materials, submitted to the impact of a metallic projectile. The design of a system with the impact side in ceramic material ( $\mathrm{SiC}$ ) was made, with a support of an ultra-high molecular weight polyethylene (UHMWPE), plaques made with $200 \mathrm{~mm}$ square geometry and $5 \mathrm{~mm}$ thick, and polymeric tissue cuts with equal area dimensions. The (NIJ) Standard 0101.06 was taken as reference for testing the ballistic impact essay; crater formation was identified in the ceramic material; using optical microscopy and scanning fractures and delamination were identified as predominant failure mechanisms; in the polymeric material, fibrillation twist, fiber fusion, conical deformation and delamination were observed and then samples were analyzed using "Morye, Smith and Retch e Ipson" kinetic energy balance models. The result showed that the most influential to dissipate energy, is the panel made with dual ceramic plate ( $\mathrm{SiC}$ ) and double-layer polymeric tissue as a backup.

Keywords: composite systems, impact, failure mechanisms, kinetic energy dissipation.

\section{INTRODUCCIÓN}




\subsection{Mecanismos de falla más representativos}

La creciente demanda de medios para protección balística ha incrementado la exploración en el diseño y el desarrollo de materiales aplicables en sistemas de protección vehicular, aeronáutica y personal, entre otras. En este campo de la ingeniería es imprescindible la identificación de las propiedades de los materiales y los mecanismos de falla de los sistemas empleados, así como el reconocimiento de los factores que predominan en el análisis de disipación de energía.

Según Zukas [1]], se entiende por impacto balístico, a la colisión entre un proyectil y un objeto, llamado también objetivo. Para el estudio de absorción de energía se deben considerar algunos factores importantes, uno de los cuales es la velocidad de deformación, se ha demostrado que a medida que aumenta la velocidad del impacto, la forma de la estructura del objetivo, es secundaria, frente al comportamiento del material; a velocidades de impacto relativamente bajas, menores a $10 \mathrm{~m} / \mathrm{s}$, la forma y la rigidez de la estructura resultan dominantes, pero cuando la velocidad de impacto supera los $300 \mathrm{~m} / \mathrm{s}$, las propiedades mecánicas del material controlan la respuesta del objetivo, es así, que si el impacto tiene velocidades muy elevadas (mayores de $1000 \mathrm{~m} / \mathrm{s}$ ), el proyectil se considera en el umbral que se conoce como hipervelocidad [1 $\underline{2}, \underline{3}, \underline{4}$; ; en esta clasificación el material se comporta como un fluido sometido a altas presiones.

Se ha observado que la energía cinética de los proyectiles es absorbida en el acero a través de mecanismos de deformación plástica, evidenciada por la formación de un cráter, fisuras, delaminación, trasferencia de calor y, dependiendo del espesor de la placa, con la formación de un cono []].

En los materiales cerámicos se produce erosión, grietas y formación de un cráter durante el frenado del proyectil por un impacto balístico; la fragilidad de este material es una limitante para su utilización, por lo que debe ser utilizado unido a otro material, por ejemplo, una placa metálica o varias capas de tejido polimérico que absorban la energía cinética residual. La Figura 1 muestra los mecanismos de falla más representativos en sistemas cerámicos, activados por el impacto de un proyectil en placas cerámicas [ㅁ, 7]]. Se destacan tres modos de falla característicos: un modo frágil, que origina crecimiento de microgrietas entorno al cráter; uno radial, que produce clivaje alrededor del cráter; y otro de fragmentación, que conduce a la rotura completa del material. Estos modos son determinados por las propiedades del material, el proceso de manufactura y el diseño de la placa []].
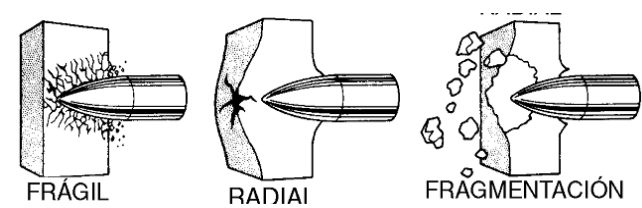

Figura 1: Modos de falla producidos en placas cerámicas, durante el impacto de proyectiles []].

Algunos materiales poliméricos tienen propiedades mecánicas altas que, sumadas a su baja densidad, los hace aptos para el diseño de sistemas de protección personal. Los mecanismos de falla observados en este grupo de materiales luego de un impacto balístico son: deformación, fusión, torsión, rotura de fibras y formación de un cono. Los mecanismos de falla en materiales poliméricos están relacionados con las características del tejido y la tensión ejercida sobre la fibra está en función de la distancia del impacto, pues los hilos que están más cerca experimentan tensión alta, de falla, mientras que los hilos más distantes del punto de impacto son sometidos a la deformación cónica del tejido, no están sometidos a corte []ㅡ, ver Figura 2. 


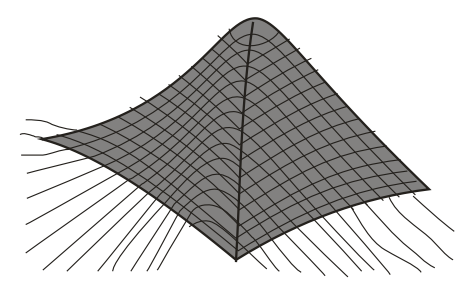

Figura 2: Efecto de formación cónica en tejidos de aramida

\section{2 DISIPACION DE ENERGIA CINETICA}

\section{2.2. Relación de mecanismos de falla y absorción de energía en materiales cerámicos y poliméricos.}

Para realizar el estudio del balance de energía se tienen en cuenta las siguientes características del sistema compuesto, para el material cerámico: masa, densidad y dimensiones del sistema; masa, diámetro y velocidad del proyectil, velocidad residual del impacto y la masa del tapón (material eyectado producto del impacto).

En los materiales poliméricos la absorción de energía cinética se rige por las propiedades del material, la forma del tejido y el espesor del sistema, las cuales determinan el tamaño de la deformación cónica en la cara posterior del tejido, y la energía absorbida por delaminación no se tiene en cuenta. La reducción de velocidad y retención de fragmentos ocurre a través de mecanismos de deformación elastoplástica de la fibra, en sentido transversal y longitudinal $[\underline{9}, \underline{10}]$. El balance de energía realizado para estudiar el impacto de un proyectil sobre un sistema de protección, puede obtenerse a partir de la diferencia entre la energía incidente (energía absorbida por deformación en la zona de corte del cráter $(E o)$ ) y la energía residual del proyectil, la cual corresponde a la energía perdida por calor y deformación durante la perforación debido a la presencia de una zona de corte circunferencial entorno al impacto $(E r)$ con la masa del mismo. Ver ecuaciones 1 y 2 donde $V o$ y $V r$ son las velocidades inicial y residual del proyectil respectivamente; mp es la masa del proyectil y ms es la masa del tapón, es decir, la masa desalojada del panel por el proyectil durante el impacto [11].

$$
\begin{aligned}
& E_{0}=\frac{1}{2} \cdot m \cdot V_{0}^{2} \\
& V_{r}=\left(\frac{m p}{m p+m s}\right) \cdot V_{s}
\end{aligned}
$$

La absorción de energía [11] se puede expresar como:

$$
\Delta E=E_{0}-E_{r}=\frac{1}{2} \cdot m \cdot v_{0}^{2}-\frac{1}{2} \cdot m \cdot V_{r}^{2}=\frac{1}{2} \cdot m \cdot\left(v_{0}^{2}-v_{r}^{2}\right)
$$

Para analizar la disipación de energía en materiales poliméricos, Smith [9] propuso un modelo que analiza la incidencia del proyectil sobre un único hilo del tejido y concluyó que un hilo impactado perpendicularmente es sometido a la tensión de dos ondas que viajan a lo largo del mismo: una longitudinal que viaja a la velocidad del sonido e inicia el movimiento de las partículas en el hilo hacia el área de impacto, y otra denominada 'transversal', la cual es más lenta que la longitudinal, que origina el movimiento de las partículas del hilo en la misma dirección del proyectil. En un segundo modelo, planteado por Morye [10], concebido para estudiar sistemas tejidos laminados heterogéneos (por ejemplo, en sistemas tipo sándwich), la energía absorbida por el sistema durante el impacto es igual a la energía cinética pérdida por el proyectil. Se consideran cinco mecanismos de deformación que contribuyen a la disipación de energía proveniente del proyectil, los cuales son: $\mathrm{E}_{\mathrm{T}}$ (energía total absorbida por el laminado de material compuesto), EFP (energía absorbida por el fallo de las fibras primarias), EFS (energía absorbida por la deformación elástica de las fibras secundarias), $E K C$ (energía absorbida en la formación y movimiento del cono en la cara posterior del panel), $E D L$ (energía absorbida debido a la delaminación del material) y ERM (energía absorbida debido a la rotura de la matriz), las cuales se aplican en el siguiente modelo:

$$
\mathrm{E}_{\mathrm{T}}=E_{F p}+E_{F s}+E_{K C}+E_{D L}+E_{R M}
$$

Para modelar la absorción de energía cinética por el mecanismo de formación del cono $\left(\mathrm{E}_{\mathrm{KCi}}\right)$ en el 
lado posterior del laminado, se encontró que los vértices formados durante la perforación se mueven a la misma velocidad del proyectil $(\mathrm{Vi})$, y la profundidad de la misma es igual al desplazamiento total del proyectil durante el contacto. Con esta información se puede determinar la disipación de energía usando la siguiente ecuación:

$$
E_{K C_{i}}=\frac{1}{2} \cdot m_{c_{i}} \cdot V_{i}^{2}
$$

Donde, $\left(M_{C_{i}}\right)$ es la masa del cono que se forma en la cara posterior de la placa de material compuesto.

En un tercer modelo semiempírico aplicado para hallar el balance de energía, presentado por Retch e Ipson $[\underline{12}, \underline{13}]$, se halla la energía disipada por la generación del cráter durante el impacto (Ecuación 6), y para realizar el balance de energía se tienen en cuenta las siguientes características del sistema compuesto: masa, densidad y dimensiones del sistema; masa, diámetro y velocidad del proyectil, velocidad residual del impacto y la masa del tapón $[\underline{13}, \underline{14}]$ se presentó la siguiente fórmula de balance de energía:

$$
E T=E+W
$$

Donde $E T$ es la energía total absorbida, $W$ es la energía absorbida por deformación en la zona de corte del cráter y $E$ es la diferencia entre la energía cinética inicial y la energía residual, la cual corresponde a la energía perdida por deformación y calor durante el impacto; la energía cinética adicional (w) puede ser perdida a través de la deformación durante la perforación debido a la presencia de la zona de corte circunferencial y el balance queda así:

$$
\frac{1}{2} m p \mathrm{~V}_{\mathrm{S}}^{2}=\mathrm{E}+\mathbf{W}+1 / 2(\mathrm{mp}+\mathrm{ms}) \mathrm{Vr}^{2}
$$

Para obtener la velocidad residual durante la formación del cráter:

$$
\mathrm{V}_{\mathrm{r}}=\left[\frac{\mathrm{mp}}{\mathrm{mp}+\mathrm{ms}}\right] \mathrm{Vs}
$$

Donde: $V r$ es la velocidad residual, $V s$ es la velocidad inicial, $m p$ es la masa del proyectil y $m s$ es la masa del tapón. Este modelo brinda información valiosa para realizar el análisis a los sistemas en exploración predominantemente rígidos y puede ser complementado con el modelo presentado por Smith, asumiendo que el respaldo flexible de polietileno (UHWMPE) permanece unido a la cerámica como una membrana circular con una masa inicial que aporta al sistema reduciendo la velocidad del proyectil durante el impacto.

\section{TRABAJO EXPERIMENTAL}

El objetivo de este trabajo de investigación es el de encontrar y analizar los mecanismos de falla en sistemas compuestos tipo sándwich, utilizando placas de carburo de silicio y láminas de polietileno de ultra alto peso molecular, impactados por la cara cerámica con un proyectil metálico.

\subsection{Sistema Estudiado}

En este trabajo se exploró un panel laminado compuesto a partir de materiales poliméricos flexibles (UHMWPE) y materiales rígidos, como lo son los cerámicos (SiC). Se realizó el diseño de un sistema con la cara de impacto en material cerámico con respaldo elaborado en polietileno de peso molecular ultra alto, con las siguientes configuraciones ilustradas en la Figura tres (3), (cerámica/polietileno; doble cerámica/polietileno; cerámica/doble polietileno; doble cerámica/doble polietileno). Las placas fueron elaboradas con geometría cuadrada de $200 \pm 5 \mathrm{~mm}$ de lado y $5 \pm 1 \mathrm{~mm}$ de espesor, y módulos cuadrados de tejido polimérico cortados de $200 \pm 5 \mathrm{~mm}$ de lado. 


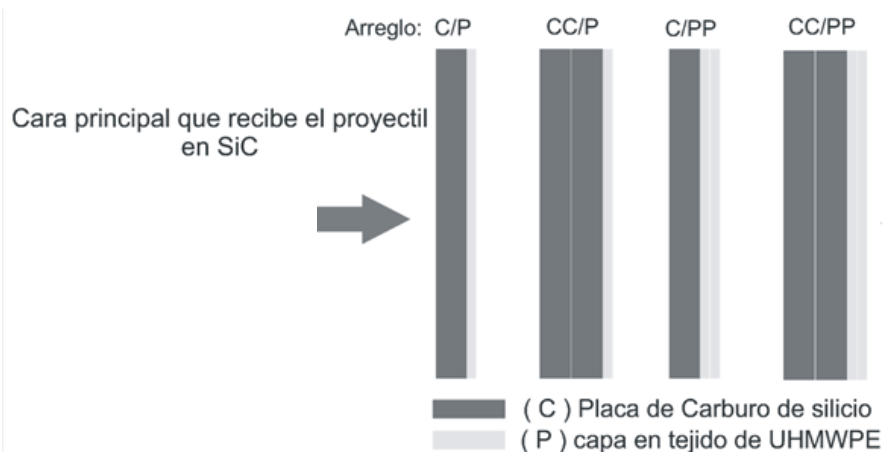

Figura 3. Representación gráfica de las configuraciones utilizadas

\subsection{Materiales}

La selección de los materiales para la fabricación del panel s realizó a partir del conocimiento de las propiedades de polímeros y cerámicos empleados en aplicaciones balísticas. Como componente flexible se seleccionó el tejido laminado de polietileno de ultra alto peso molecular (UHMWPE) debido a su alta capacidad de absorción de energía cinética y como material cerámico se seleccionó al carburo de silicio (SiC) dado que es un material con alta dureza, alta resistencia a la abrasión y ha sido ampliamente utilizado en aplicaciones balísticas $[\underline{15}, \underline{16}]$. Para determinar la porosidad de la cerámicas utilizadas, estas se secaron y sinterizaron en horno a aproximadamente $850^{\circ} \mathrm{C}$ y posteriormente se ensayaron de acuerdo con la norma ASTM C1039 [15]. Se realizó un análisis de difracción de rayos X (DRX) para identificar el cerámico empleado. En el Cuadro 1 se observan las características y las propiedades de la placa cerámica obtenida.

Tabla 1: Propiedades del carburo de silicio empleado

\begin{tabular}{l|l}
\hline PROPRIEDADES DEL MATERIAL & CARACTERÍSTICAS DE LA PLACA \\
\hline Cerámico: Carburo de silicio fase alfa & \\
\hline $\begin{array}{l}\text { Tamaño de grano: pureza del material: } 80 \% \text { Silicio, } \\
20 \% \text { sílice y aglomerante a base de celulosa }\end{array}$ & $\begin{array}{l}\text { Conformado por compactación a presión atmosférica } \\
\text { y sinterizado en horno eléctrico a } 850{ }^{\circ} \mathrm{C}\end{array}$ \\
\hline Porosidad aparente: $40 \%$ & Densidad teórica del SiC $: 3.1-3.2 \mathrm{~g} / \mathrm{cm}^{3}[16]$ \\
\hline Densidad real media: $1.60 \mathrm{~g} / \mathrm{cm}^{3}$ & Masa media de la placa: $293.4 \mathrm{~g}-312.9 \mathrm{~g}$ \\
\hline
\end{tabular}

El adhesivo utilizado para adherir la placa cerámica con el tejido polimérico fue resina epóxica, debido a que tiene muy buena adherencia en materiales no homogéneos[18]. Algunas de las propiedades de la resina epóxica utilizada en éste trabajo son: densidad: $1.1-1.4 \mathrm{~g} / \mathrm{cm}^{3}$, temperatura de transición vítrea $\left({ }^{\circ} \mathrm{C}\right)$ : 120 - 190, viscosidad: 113 centipoise a $22{ }^{\circ} \mathrm{C}$. Baja retracción, buen comportamiento mecánico hasta $180^{\circ} \mathrm{C}$, buena resistencia a agentes químicos, alta resistencia al desgaste y alta resistencia al agrietamiento.

\subsection{Métodos}

Para la detección y el análisis de los mecanismos de falla en los sistemas planteados se procedió a observar la geometría, cortes y deformaciones superficiales y transversales en cada uno de los arreglos. Primero se realizó la medición utilizando un calibrador pie de rey para determinar el tamaño del diámetro del cráter de entrada, el diámetro del cráter de salida y el diámetro del cono en la contracara de cada uno de los arreglos. Para realizar las secciones transversales a cada arreglo se utilizó hidrocorte y posteriormente se realizó un balance de energía para observar las diferencias de disipación de energía entre los arreglos experimentales.

Para el diseño experimental se determinaron tres factores potenciales de diseño: uno correspondiente a la combinación originada y dos factores correspondientes al tipo de material que recibe el impacto [19, 20]; como variables respuesta fueron seleccionados el diámetro del cráter de entrada y en la contracara los diámetros del cráter de salida y del cono.

Se determinó un impacto para cada arreglo en la realización de la prueba balística, siguiendo el 
protocolo del numeral 4.0 de la NIJ (National Institute of Justice de EE.UU.) [21]. El proyectil calibre 9mm utilizado tiene forma semiesférica, $8 \mathrm{~g}$ de masa, con una velocidad de impacto de $380 \pm 3 \mathrm{~m} / \mathrm{s}$. Durante esta exploración se excluyó la medición de la profundidad de las perforaciones sobre el backing, debido a que no eran el objeto de este estudio. No fue posible medir la velocidad residual debido al montaje diseñado para la norma, el cual se ilustra en la Figura 4. Se observaron las diferencias en los mecanismos de falla utilizando medición y observación de las fracturas superficiales utilizando fotografía digital y microscopia óptica, y para ver los detalles más profundos se aplicó microscopia electrónica de barrido SEM, de forma aleatoria a uno de los arreglos que presentó el efecto principal y la interacción predominante en el diseño experimental.

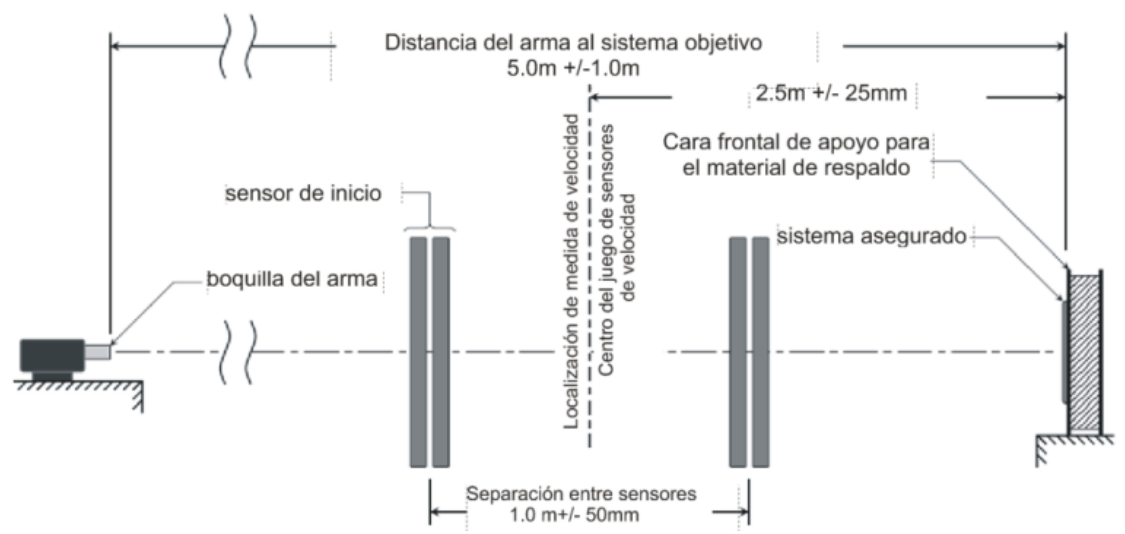

Figura 4: Montaje requerido para la realización de pruebas de impacto balístico según la norma NIJ

\section{RESULTADOS Y DISCUSIÓN}

\section{1 Mecanismos de Falla}

En las Figuras 5 a la 9 se muestran las imágenes más representativas del sistema cerámico/polímero, en las cuales se pueden identificar los mecanismos de falla específicos para cada uno de los arreglos.

En la Figura 5, se puede observar el corte transversal del arreglo C/P, con la formación de una fractura que inicia la propagación a partir del borde del cráter hacia el interior de la placa cerámica en forma simétrica hasta presentar astillamiento (spalling) alrededor del cráter formado; esta grieta se propaga sólo por el cerámico hasta el borde del arreglo.

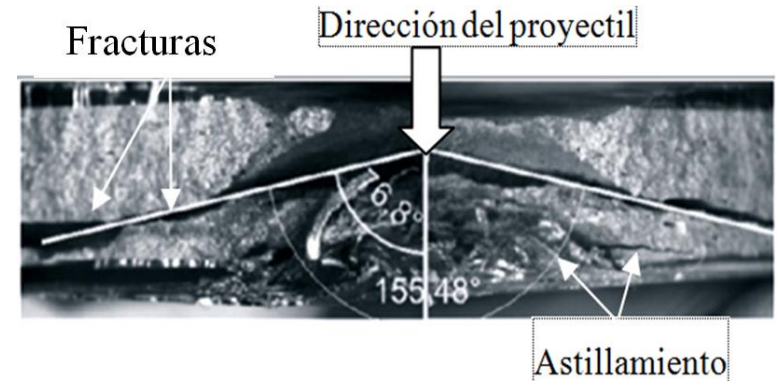

Figura 5: Corte transversal del arreglo C/P.

En la Figura 6, imagen B, se presenta la falla del arreglo CC/P y se puede ver el daño originado sobre el componente cerámico en dirección de la penetración del proyectil; asimismo, se ilustran mecanismos de fractura a una delaminación y desprendimiento del material (spalling), producto de las ondas de tensión transversales durante el impacto. También se observan algunas grietas propagadas transversalmente que fracturan al sistema de forma paralela a la superficie y se observa la rotura de las fibras en la capa de tejido polimérico ilustradas en la fotografía $\mathrm{C}$. 


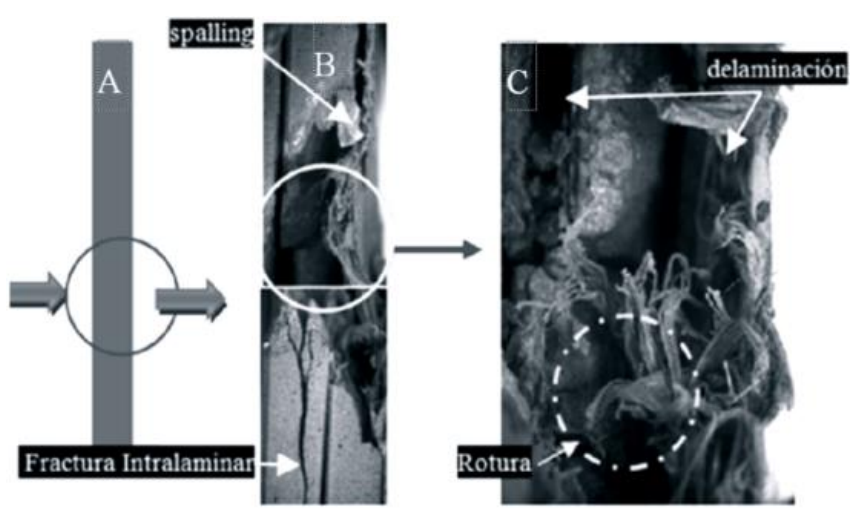

Figura 6: Mecanismos de falla en el arreglo CC/P.

En la Figura 7, imagen A, se observa en un corte transversal del arreglo CC/PP, delaminación y un leve levantamiento del cerámico (fragmentación) y en la fotografía B se observa delaminación entre las capas del tejido, junto a una de las fracturas donde se pudo ver que el desprendimiento de material fue perpendicular a la trayectoria del proyectil.
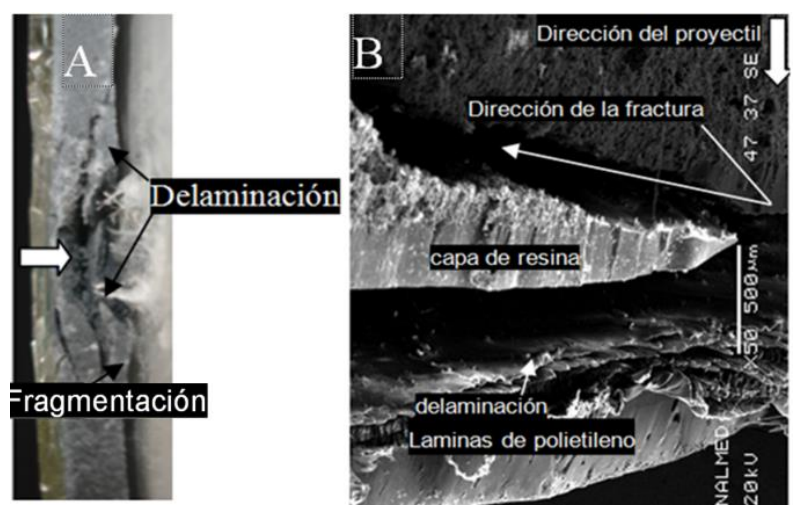

Figura 7: Mecanismos arreglo CC/PP.

En Figura 8, imagen A, se pueden ver el cráter, la delaminación y cinco cortes distribuidos en la tela entorno al centro del impacto (líneas de color rojo) generado por el proyectil en la contracara del arreglo. En la imagen B se observa un vacío entre las placas cerámicas originado en la capa adhesiva de resina, dicho vacío funcionó como mecanismo inhibidor de la propagación de la fractura, que al no encontrar un sólido para ser propagada termina por desaparecer.
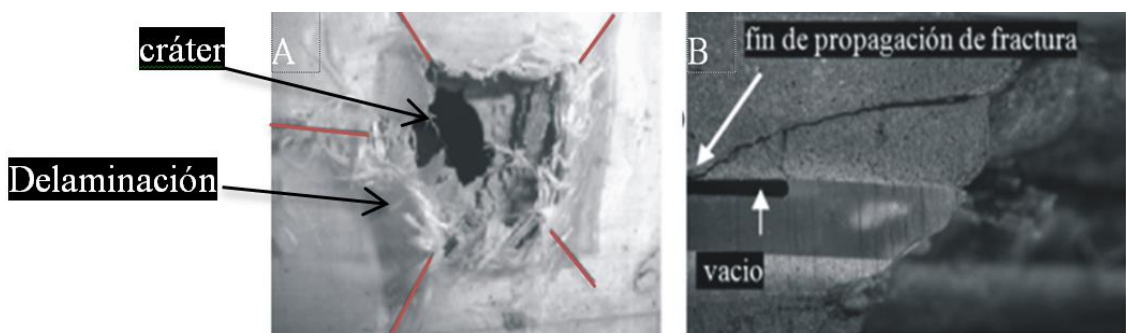

Figura 8. Detalles de mecanismos de falla en arreglo CC/PP.

En la Figura 9, imagen A, se puede ver el cráter formado en la vista superior del sistema y mediante un acercamiento al cráter se observa la rotura del tejido (fibras primarias), junto a una marca circular en el tejido polimérico en torno al cono. En la imagen B se ilustra el cono, la formación de un cuello (necking) y la deformación en forma de pétalos (petalling) como resultado del contacto directo con el proyectil 


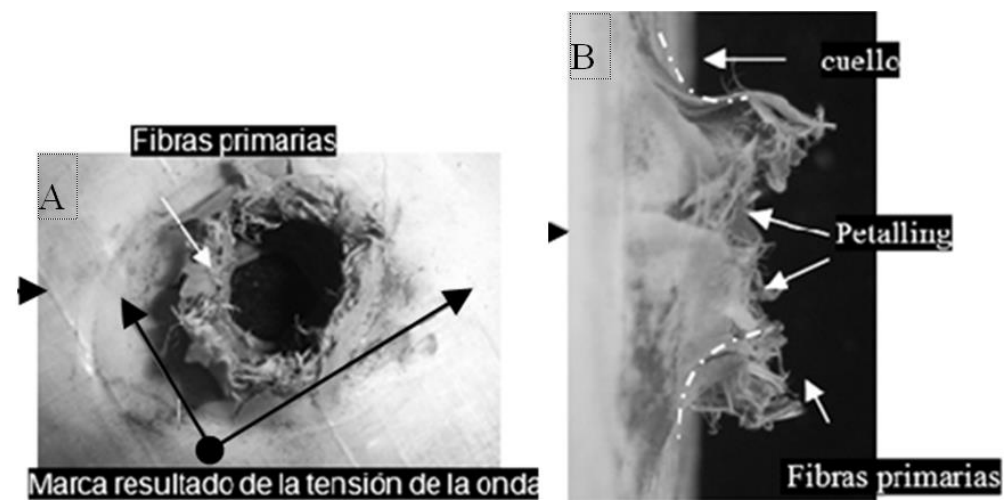

Figura 9: Detalles de mecanismos de falla en la cara posterior del arreglo CC/PP.

\section{2 Resultados en el análisis de Absorción de Energía}

Se realizó un balance de energía para observar las diferencias de disipación de energía entre los arreglos experimentales. Se decidió complementar el análisis de Retch e Ipson donde se estudian la masa del proyectil y del material desalojado del sistema durante el impacto, sumando a este estudio el balance realizado por el equipo de Morye que presenta una aproximación a la absorción de energía en un material tejido, considerando la formación de un cono en la cara posterior de un sistema.

Se observó que el factor que mejor absorbió la energía fue de carácter rígido y el principal mecanismo de disipación estuvo en la formación de cráteres; mientras que el componente flexible del sistema, ubicado en la contracara, presentó como mecanismo de falla la deformación del tejido con geometría cónica; en este modelo se consideró como energía perdida la asociada con la fricción, la generación de grietas, la rotura de fibras y la trasmisión de calor. De este modo se presenta un modelo general con los mecanismos de falla observados en la investigación. El modelo aplicado resultó de la siguiente forma:

$$
\mathrm{ET}=\mathrm{E}+\mathrm{W}+\mathrm{Ekci}
$$

El Modelo de Retch e Ipson, complementado con el análisis realizado por la formación cónica en el material flexible de Morye et al. (donde ET es la energía total absorbida, E es la diferencia entre la energía cinética inicial y la energía residual), esta corresponde a la energía perdida por deformación y calor durante el impacto y W es la energía absorbida por deformación en la zona de corte del cráter y Ekci es la deformación del material flexible con geometría cónica.

En la Figura 10 se observa en la columna horizontal los datos de la cantidad de energía disipada por cada sistema y en la fila horizontal se muestran los arreglos experimentales; se evidencia una gran diferencia entre la energía disipada entre los arreglos sencillos(C/P) frente a los arreglos con doble placa de material cerámico (CC/PP). Los datos que se obtuvieron luego de aplicar el Modelo de Retch e Ipson y de Morye et al, se exponen en párrafos anteriores.

Resultado de los impactos en los sitemas analizados:

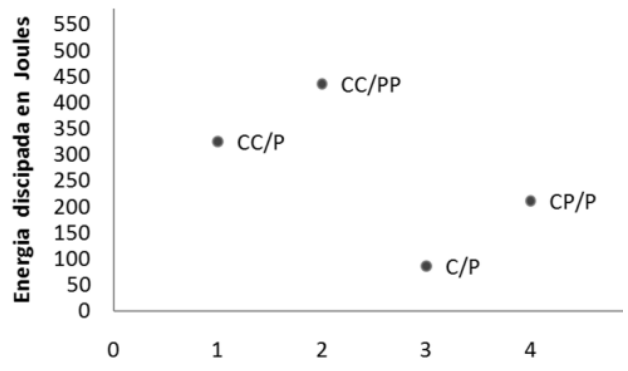

Figura 10: Resultados comparativos del valor medio de la energía disipada (Joules). 


\section{CONCLUSIONES}

La Fractografía hallada en el componente cerámico está definida en la formación de un cráter, erosión y fractura, mientras que en el componente polimérico se observó delaminación, formación de cono, pétalos, rotura y fusión en las fibras.

Se observó mayor número de mecanismos de falla en los arreglos dobles (CC/PP) tales como fragmentación y erosión en los cerámicos, pétalos, delaminación y cortes en la tela polimérica, datos que se evidencian en las tomas fotográficas.

\section{RECOMENDACIONES}

Para un análisis más juicioso de experimentos con impacto balístico, se recomienda hacer un montaje para la toma de datos de tal forma que se ubiquen sensores de velocidad en la parte posterior de las placas, con el objetivo de tomar velocidades residuales y hacer más precisos los análisis de disipación de energía cinética.

\section{AGRADECIMIENTOS}

Es necesario expresar un especial agradecimiento a la Comercializadora Internacional Grupo Empresarial Caballero SAS, en cabeza del ingeniero Miguel Caballero, por su decisiva colaboración, disposición y financiación de las pruebas balísticas, laboratorios y material polimérico. Se agradece también a la empresa Quimiresinas, seccional Bogotá, por el aporte de la resina epóxica y hago manifiesta mi gratitud hacia el Alma Mater, al laboratorio de Metalografía y ensayo de materiales de la Universidad Nacional de Colombia, sede Medellín, por aportar los recursos para la adquisición de las placas cerámicas utilizadas en la realización de la exploración.

\section{BIBLIOGRAFÍA}

[1]ZUKAS J., Impacts dynamics, 1ed., California, Krieger Pub.co, 1982.

[2] GALVEZ, F.D., Caracterización mecánica de materiales cerámicos avanzados a altas velocidades de deformación, Tesis de D.Sc, Universidad Politécnica de Madrid, Madrid, España, 1999.

[3] CUNNIFF, P.M., AUERBACH, M.A., High performance M5 Ballistics fiber/structural composites, In: Report MA 01760-5019, U.S. Army Soldier and Biological Chemical Command, Arnhem, the Netherlands 1999.

[4] KANG, T., CHEOL, K, "Energy-absorption mechanisms in Kevlar multiaxial warp-knit fabric composites under impact loading”, Composites Science and Technology, v. 60, n.5, pp.773-784, Nov. 2000.

[5] . ZHEN, L, LI, G.A., ZOU, D.L., et al., "Characterization of the deformed microstructure in 1Cr18NiTi stainless steel under ballistic impact”, Materials Science and Engineering: A, v. 489, n.1-2, pp. 213-219, Aug. 2008.

[6] BRANDON, D.S., "The ballistic faillure mechanisms and sequence in semi- infinite Supported alumina Tiles”, Materials Research, v. 12, n.5, pp. 1335-1343, May.1997.

[7] DÍAZ-RUBIO, F.G, Caracterización mecánica de materiales cerámicos avanzados a altas velocidades de deformación. Tesis de D.Sc., Universidad Politécnica de Valencia, Valencia, España, 1999.

[8] MEDVEDOVSKI, E. "Ballistic performance of armour ceramics: Influence of design and structure. Part 2", Ceramics International, v. 36, n.7, pp. 2117-2127, Sept. 2010.

[9] SMITH, J.C., "Stress strain relationships in yarns subjected to rapid impact loading, transverse impact tests", Journal of research of the national bureau of standars, v. 57, n.2, pp.83-89, Aug. 1956.

[10] BHATNAGAR, A., "Lightweight ballistic composites, military and law-enforcement applications" 1 ed., Washington D.C. USA, Crc Press, 2006.

[11] MORYE, S. S, HINE, P. J, DUCKETT, R. A, et al., "Modeling of the energy absorption by polymer composites upon ballistic impact", Composites Science and Technology, v. 60, n.14, pp.2631-2642, Nov. 2000. 
[12] CHOCRON, B. J., "Simple analytical model for ballistic impact in composites", Journal of Physique, v. 67. n. 7, pp. 821 - 826. Jul.1997.

[13] BHATNAGAR, A., "Lightweight ballistic composites, military and law-enforcement applications". 1 ed., Washington D.C. USA, Crc Press, 2006.

[14] HAZELL, P.J., APPLEBY-THOMAS, G., "A study on the energy dissipation of several different CFRP-based targets completely Penetrated by a high velocity projectile", Composite structures, v. 91, n.1, pp. 103-109, May 2009.

[15] VARGAS, F., LÓPEZ, E., LÓPEZ, D., et al., "Fabricación de tubos de carburo de silicio para uso como cuerpo radiante en hornos y sistemas de calentamiento", Dyna, v. 76, n. 157, pp. 233 - 242. Mar. 2009.

[16] WESEL J., "Handbook of advanced materials: advanced ceramics materials", 1 ed., New Jersey, USA, John Wiley and Sons, 2004.

[17] BHATNAGA, A., "Lightweight ballistic composites, military and law-enforcement applications", 1 ed. Washington D.C. USA, Crc Press, 2006.

[18] TEXTOSCIENTIFICOS.COM, http://www.textoscientificos.com/quimica/adhesivos, accedida en abril de 2011.

[19] MONTGOMERY, D.C., "Diseño y Análisis de Experimentos”, 2 Ed., Mexico D.F., Limusa Wiley. 2007.

[20] PULIDO, H. G, SALAZAR, R., CARRASCO, A. C., et al., "Análisis y diseño de experimentos”, 2 ed., Mexico D.F., Mc Graw Hill, 2008.

[21] BALLISTIC RESISTANCE OF BODY ARMOR, http://www.nij.gov/pubs-sum/223054.htm, accedido en octubre de 2013. 